\title{
A Reductive Analysis of Statements about Universals
}

\author{
Ben White
}

Forthcoming in Synthese

\begin{abstract}
This paper proposes an analysis of statements about universals according to which such statements assert nothing more than that the evidence we'd take to confirm them obtains, where this evidence is understood to consist solely of patterns in the behavior of particulars that cannot be explained by other regularities in the way things behave. On this analysis, to say that a universal exists is simply to say that there is such a pattern in the behavior of certain particulars, and for any predicate $\mathrm{F}$ that is presumed to correspond to a universal, to say that a particular is F is simply to say that its behavior exhibits a pattern of this sort. I argue that there is no theoretical work that we want postulations and ascriptions of universals to do that they'd be unfit for if analyzed in this way, and consequently that there is no reason to treat such statements as asserting anything more than what the proposed analysis suggests.
\end{abstract}

Keywords: universals; laws of nature; properties; nominalism; quidditism.

\section{Introduction}

This paper proposes an analysis of statements postulating universals or ascribing them to particulars (henceforth simply "statements about universals") according to which such statements assert nothing more than that the evidence we'd take to confirm them obtains, where this evidence is understood to consist solely of basic regularities in the behavior of particulars (i.e. regularities that cannot be explained by other regularities in the way things behave). On this analysis, to say that a universal $\mathrm{F}$ exists is merely to say that there is a basic pattern in the behavior of certain particulars (viz. those we designate as Fs), and for any predicate F that is presumed to correspond to a universal, to say that a particular is $\mathrm{F}$ is merely to say that its behavior exhibits such a pattern. ${ }^{1}$

\footnotetext{
${ }^{1}$ The terms "pattern" and "regularity" are used interchangeably throughout. I'll also speak interchangeably of the behavior of certain particulars as "exhibiting" or "manifesting" a regularity R, by which I mean merely that their behavior provides positive instances of $\mathrm{R}$ (or, alternatively, that if there were no particulars that behaved as the particulars whose behavior "exhibits" or "manifests" $\mathrm{R}$ do, we'd have no reason to believe that R exists).
} 
The structure of the paper is as follows: Section 2 suggests that the evidence on the basis of which we postulate universals and ascribe them to particulars consists of basic patterns in the way things behave. Section 3 offers an analysis of statements about universals according to which they commit us to nothing more than the existence of such patterns, and discusses the implications this analysis has for quidditist conceptions of universals. Sections 4 distinguishes two different ways of analyzing statements in terms of the evidence we'd take to confirm them, clarifies which of these two types of analyses my proposed analysis belongs to, and distinguishes two different ways of developing the analysis, one of which leads to nominalism and the other to a form of realism I call reductive realism. ${ }^{2}$ Section 5 then defends the analysis via the Occamist argument that there is no reason for interpreting statements about universals as affirming the existence of anything more than the regularities that serve as evidence for them.

\section{On what basis do we postulate and ascribe universals?}

A common strategy in philosophical analysis is to start by examining the evidential basis of certain statements and then use this to support one's theory about the content of such statements and the nature of the things that they purport to be about. ${ }^{3}$ This methodology can be particularly useful in dealing with statements about theoretical entities, where neutral means of

\footnotetext{
${ }^{2}$ A precedent for this view might be found in Alexander (1920, pp.208-32), who similarly describes universals as "habits," "laws," "patterns," or "plans of configuration" of space-time, and holds that "particulars are complexes of space-time and belong therefore to the same order or are of the same stuff as the universals which are plans of space-time." Many thanks to an anonymous reviewer for bringing this to my attention.

${ }^{3}$ Here I have in mind such examples as behaviorist and functionalist analyses of psychological statements in terms of stimuli and behavioral responses (Carnap 1932; Lewis 1972), analyses of statements about material objects in terms of sensory experiences or sense-data (Berkeley 1710/1982; Russell 1914; Ayer 1936, chap.3), Hume's (1748/2007) analysis of statements about causal relations in terms of constant conjunctions, and (more generally) empiricist analyses of statements containing theoretical terms in terms of the observational evidence on the basis of which such statements are made. While analyses of this sort have often been framed in terms of observational evidence, this is, I think, inessential to the general strategy at issue. I thus see no reason why non-observational knowledge couldn't serve as evidence for certain types of statements which turn out to be analyzable in terms of that evidence. If my proposed analysis is sound, some of the patterns of behavior that statements about universals are to be analyzed in terms of may qualify as non-observational evidence of this sort.
} 
deciding between alternative analyses are particularly hard to come by. Here it can be helpful to constrain our theorizing about the nature of such entities by requiring some justification for any ontological commitments that go beyond the evidence we'd take to confirm the statements we make about them. Such an approach may consequently prove beneficial in developing a theory of universals. Here too we might hope to better understand what statements about universals commit us to, and what the nature of universals is, by looking at the evidence that we use to confirm such statements, and then asking whether we must interpret them as asserting anything more than that that evidence obtains if they are to be capable of doing certain theoretical work that we want them to do.

To simplify matters, I'll assume throughout (and solely for purposes of exposition) that our everyday talk about various properties that particulars possess and share in common with other particulars involves a pre-theoretical commitment to realism about universals. Nothing hangs on this, for as discussed in Section 4, the analysis of statements about universals that I'll be proposing is compatible with both realism and nominalism. I therefore take no stance in this paper on whether properties should be conceived as universals or classes of resembling particulars. On the assumption that our everyday talk is conducted from a realist standpoint, the nominalist interpretation of my analysis provides a way of translating our everyday statements about universals into a nominalist language, but one could just as easily drop this assumption, and instead hold that the kinds of statements being analyzed are pre-theoretically taken to be merely about classes of resembling particulars.

Assuming, then, that we are (at least pre-theoretically) realists, and that we take certain of the properties that we postulate and ascribe to particulars to be genuine universals, on what evidence do we make such statements about universals, or, given any such statement, what sort of evidence would we take to confirm it? I suggest that this evidence consists of regularities in the behavior of particulars that cannot be explained by other regularities. I'll call such 
regularities basic regularities. Upon encountering a basic regularity of this sort, we postulate a universal that the particulars whose behavior exhibits that regularity share in common. The particulars to which this universal is ascribed are thereby grouped together as members of a class consisting of all and only those things whose behavior exhibits the regularity in question. While universals might sometimes be postulated on the basis of a single observation of a particular behaving in a novel and unexplainable way, we do so only on the assumption that the instance we've observed is part of a more general pattern that could (at least in principle) have other instances.

In saying that universals are postulated on the basis of basic regularities in the way things behave, I am using the term "behavior" quite broadly to include any kind of change or lack thereof in a particular's motion, state, or relations to other particulars, including any effect it has on the motion, state, or relations of other particulars. Any way in which a particular is disposed to undergo or resist such changes, or produce or prevent such changes in other particulars, will thus be described as a pattern or regularity in its behavior. ${ }^{4}$ Any particulars that are disposed to undergo, resist, produce, or prevent the same kinds of changes in the same conditions will accordingly be said to exhibit the same pattern in their behavior.

This broad notion of behavior helps deal with putative universals like colors or shapes, which some might think we ascribe to particulars not on the basis of how they behave but instead simply on the basis of our perception of similarities in their intrinsic qualities, or how they intrinsically are. Note that on the notion of behavior I'm employing, the fact that certain particulars produce similar perceptual experiences in us qualifies as a pattern in the way they behave. Insofar as we postulate a universal of redness that is instantiated by external objects, we thus do so on the basis of regularities in the way red objects behave in various illumination

\footnotetext{
${ }^{4}$ This may sound like dispositional essentialism, but it's not. I'll explain why in Section 4.
} 
conditions, e.g. by reflecting, transmitting, or emitting light with certain spectral characteristics and/or producing certain visual experiences in us. By contrast, if we treat redness as a universal instantiated by visual experiences, our postulation of it will instead be based on regularities in the way that red experiences behave, e.g. by being produced by certain stimuli, by prompting individuals who have such experiences to have certain thoughts, make certain judgments, and behave in certain ways depending on the context in which the experience occurs, and by standing in various relations to other color experiences. Shape universals can be handled in a similar fashion. Insofar as we postulate a universal of triangularity, our postulation of it will likewise be based on regularities in the way triangular objects behave, e.g. by producing certain visual or tactile experiences in us and/or by interacting with other objects (including measuring instruments, e.g. protractors) in various ways.

It's worth emphasizing that the types of motions, states, and/or relations that a pattern of behavior is individuated in terms of may themselves qualify as patterns of behavior (which may or may not be basic). ${ }^{5}$ Suppose there is a class of particulars $\mathrm{P}$ whose behavior exhibits a certain pattern, which consists in their exhibiting a certain type of motion $\mathrm{M}$ whenever they're in a certain state $S$. To move in manner $M$ is to behave in a certain way, and the same may likewise be true of what it is to be in state $\mathrm{S}$. The type of motion $\mathrm{M}$ and type of state $\mathrm{S}$ will then themselves qualify as patterns of behavior exhibited, respectively, by all and only those particulars that move in manner M, or which behave in the way that is distinctive of state $\mathrm{S}$. The pattern unique to $\mathrm{P}$ will consequently be distinguished by the fact that particulars belonging to that class exhibit the pattern of behavior associated with $\mathrm{M}$ whenever they're also exhibiting the pattern of behavior associated with S. If there is no explanation for why these patterns coincide in this way in $\mathrm{P}$, then the pattern unique to $\mathrm{P}$ qualifies as basic, and thus provides the

\footnotetext{
${ }^{5}$ Thanks to an anonymous reviewer for raising this issue.
} 
basis for postulating a universal that all Ps share in common, even though this pattern consists of a certain correlation between two other patterns (viz. M and S).

Applying this to the foregoing example of redness, suppose we postulate a universal of redness that is instantiated by external objects whose behavior exhibits a certain pattern, which consists partly in their reflecting, transmitting, or emitting light with certain spectral characteristics when in certain illumination conditions. The spectral characteristics cited in the individuation of this pattern can themselves be viewed as patterns in the behavior of light, which are exhibited by all and only those rays of light that are composed of certain wavelengths standing in certain relations of relative intensity to one another. The state of having a certain wavelength and relations of relative intensity can in turn be viewed as further patterns, which are exhibited by all and only those things (e.g. light, soundwaves) that move and interact with other things (e.g. spectrometers, sound level meters, the mediums through which they propagate) in certain ways. And so on. In short, patterns often consist of correlations among other patterns. Importantly, though, such patterns can still be basic. For even if a pattern $\mathrm{P}$ consists simply of some correlation among other basic or non-basic patterns P1 and P2, there may still be no explanation for why P1 and P2 are correlated in this way in the behavior of those particulars that exhibit $\mathrm{P}$.

So much for the notion of behavior that's operative in my suggestion that we postulate universals on the basis of regularities in the way things behave. Why must these regularities also be basic? The rationale for this requirement derives from episodes in the history of science, which suggest that while patterns in the behavior of particulars are often taken to indicate that those things may share a universal in common, we don't assume that there is a distinct universal corresponding to every such pattern. Thus, despite certain patterns in the behavior of all and only those things classified as jade, the discovery that such things belong to two distinct mineral kinds (jadeite and nephrite) undermines the claim that there is a universal of being jade that all 
samples of jadeite and nephrite instantiate (Kim 1992). We refrain from postulating such a universal because the similarities among samples of jadeite and nephrite that led us to classify them as jade can be explained by regularities that make no reference to jade. While samples of jadeite and nephrite resemble one another in color, texture, hardness, and other superficial features, our knowledge of the chemical compositions of jadeite and nephrite and regularities linking different chemical compositions to different superficial features leads us to expect that jadeite and nephrite would share these features in common. We consequently have no need to postulate a universal of being jade to account for the common patterns in the way that jadeite and nephrite behave, for these patterns can be explained by regularities connecting the chemical compositions of jadeite and nephrite to the behavior that each manifests. ${ }^{6}$

However, if no such explanation were available to us, then the situation would be quite different. If no other regularity could explain why jadeite and nephrite behave so similarly despite their different chemical compositions, I suspect we would postulate a universal of being jade that all things that behave in this manner instantiate. The discovery of a pattern in the behavior of certain particulars thus only leads us to postulate a universal that those particulars instantiate when that pattern cannot be explained by other patterns we know of. ${ }^{7}$

Further support for this conclusion can be derived from cases of intertheoretic reduction. When we discover in the course of carrying out such a reduction that the regularity that led us to postulate a certain universal $U$ can be explained in terms of some more basic regularities $\mathrm{R}$, we either (a) identify $\mathrm{U}$ with some universal postulated independently on the

\footnotetext{
${ }^{6}$ The same may be true of jadeite and nephrite as well. If we can explain why all samples of jadeite share the distinguishing features of jadeite by means of more basic regularities in the behavior of their constituent particles that make no reference to jadeite, then we have no reason to postulate a universal of being jadeite either.

${ }^{7}$ Note the implications this has for natural kinds like bosons, or mammals, whose membership includes such heterogenous entities as photons, gluons, and Higgs particles, or humans, whales, and bats. As with jade, I suggest that we treat such kinds as universals if and only if there is some basic regularity in the behavior of all and only their members. Otherwise we view them (like jade) merely as classes of particulars whose membership is defined by some cluster of features, each of which may or may not itself qualify as a universal, depending on whether there is a basic regularity in the behavior of all and only the things that possess that feature. Thanks to Donnchadh O'Conaill for raising this issue.
} 
basis of $\mathrm{R}$, or (b) abandon the view that $\mathrm{U}$ is a universal. The choice between (a) and (b) is determined by how closely the predicates of the reduced theory T match up with the predicates of the reducing theory $\mathrm{T}^{*}$. When certain regularities that $\mathrm{T}$ explains in terms of some predicate $\mathrm{F}$ are explained by $\mathrm{T}^{*}$ in terms of a different predicate $\mathrm{G}$, and the relations that $\mathrm{F}$ bears to other predicates in $\mathrm{T}$ correspond to relations that $\mathrm{G}$ bears to other predicates in $\mathrm{T}^{*}$ (so that $\mathrm{F}$ and $\mathrm{G}$ play similar roles in their respective theories), we typically go the route of (a), and assert that $\mathrm{F}$ and $\mathrm{G}$ denote the same universal. However, when there is no predicate whose role in $\mathrm{T}^{*}$ corresponds to that of $\mathrm{F}$ in $\mathrm{T}$, we take this to show that there is no universal of being $F$, as the only regularities that might've led us to postulate such a universal can be explained by other regularities that make no reference to anything that could be plausibly identified with $\mathrm{F}$.

The discovery of a regularity in the way certain particulars behave thus only leads us to postulate a universal that all and only those particulars instantiate when the regularity is basic. If we find that the regularity on the basis of which a putative universal $\mathrm{F}$ was postulated is explainable by other regularities, then we revoke the claim that $\mathrm{F}$ constitutes a separate item in our ontology by either identifying $\mathrm{F}$ with a universal that was postulated on the basis of other regularities, or eliminating it entirely.

\section{The proposed analysis and its implications for quidditism}

Assuming that the evidential basis of statements about universals is as just described, what if anything can we infer from this about the content of such statements? As suggested above, we might start with the hypothesis that such statements assert nothing more than that the evidence we'd take to confirm them obtains, and then see if they must be interpreted as asserting anything more than this if they are to do certain theoretical work that we want them to do. This gives us the following analysis as default: 
In saying that a universal $\mathrm{F}$ exists, we assert nothing more than that there is a basic regularity $\mathrm{R}$ in the behavior of certain particulars (viz. those we designate as Fs), and in saying that a particular is $\mathrm{F}$ (where the predicate $\mathrm{F}$ is presumed to correspond to a universal), we assert nothing more than that there is a basic regularity $\mathrm{R}$ in its behavior that is exhibited in the behavior of all and only those particulars that we designate as Fs.

Analyzed in this way, statements about universals commit us to nothing more than the existence of basic patterns in the behavior of particulars. ${ }^{8}$ I'll henceforth refer to this analysis as A+. For ease of exposition, I'll also occasionally speak of a particular $x$ as instantiating or being an instance of a universal F. By this I mean nothing more than what is asserted by the statement " $x$ is $\mathrm{F}$," where the predicate $\mathrm{F}$ is presumed to correspond to a universal. Likewise, when I speak of someone as ascribing a universal $\mathrm{F}$ to a particular $x$, by this I mean merely that the individual in question makes a judgment or assertion of the form " $\mathrm{x}$ is $\mathrm{F}$ " (where the predicate $\mathrm{F}$ is again presumed to correspond to a universal). All talk of instantiation and ascription of universals in the previous section should be understood with these points in mind.

A+ implies that at least some forms of quidditism are false. Quidditists hold that universals are "quiddities" that have no necessary relations to other universals (besides nonidentity) or to any regularities in the behavior of their instances. There are, however, a number

\footnotetext{
${ }^{8}$ One might worry that statements about universals can't be analyzed in terms of basic regularities in the behavior of particulars, because talk of ways of behaving is universals-talk, and as such is supposed to be subject to the proposed analysis. My "counter-worry" is that the same sort of objection might be raised against any analysis, for Paradox of Analysis-type reasons. For any analysis presents the kind of talk being analyzed as equivalent to the talk in terms of which it's analyzed, so if the analysis is successful, then naturally the analysans-talk will be of the same sort as the analysandum-talk. Thus, since A+ holds that statements about universals just are statements about basic ways that particulars behave, if A+ is successful, then talk of basic ways of behaving is indeed universals-talk. To reject A+ on these grounds would, however, seem to require that one reject the very possibility of analysis, for in any successful analysis, again, the talk being analyzed is shown by the analysis to be of the same sort as the talk in terms of which it's analyzed. Thanks to an anonymous reviewer for raising this issue.
} 
of ways of interpreting this thesis, depending on which of the following claims one takes it to imply:

(Q1) A universal can have instances in two different possible worlds even if there is no common pattern in the behavior of its instances in those two worlds (qua instances of that universal).

(Q2) Two particulars can behave the exact same way in the actual world without sharing any universal in common.

(Q3) Two particulars can instantiate the same universal in the actual world even if there is no common pattern in their behavior (qua instances of that universal).

Since these three claims are independent of one another, we can distinguish at least 8 different forms of quidditism (viz. [(Q1) \& (Q2) \& (Q3)], [(Q1) \& (Q2) \& (Q3)], etc.).

A+ implies that (Q3) is false. This strikes me as correct. For it is difficult to see what grounds we could ever have for postulating a universal such as (Q3) describes, and where we have no reason to think that something exists, that is sufficient reason to think that it doesn't. A+ implies that (Q2) is false as well. This also strikes me as correct. For whereas the simplest view would be to treat all particulars that exhibit a given basic regularity as instances of the same universal (as A+ does), (Q2) implies that it is possible that some of these particulars instantiate one universal, while others instantiate another, or indeed that each instantiates a different universal. Accepting (Q2) thus leaves us unable to determine whether two particulars are instances of the same universal, even if they behave in the exact same way (Shoemaker 1980, pp.116-7; Black 2000, pp.95-6; Bird 2007, pp.76-9). Such epistemic consequences seem best avoided if possible. The fact that $\mathrm{A}+$ is incompatible with both $(\mathrm{Q} 2)$ and $(\mathrm{Q} 3)$ is thus in my view an argument in its favor. 
The compatibility of $\mathrm{A}+$ with $(\mathrm{Q} 1)$ depends on how $\mathrm{A}+$ is interpreted. In analyzing universals $^{9}$ in terms of the regularities that serve as evidence for them, $\mathrm{A}+$ implies that universals can be individuated in terms of the regularities they're postulated on the basis of. Proponents of $\mathrm{A}+$ have the option of treating these individuative descriptions as either rigid or non-rigid. The former option is incompatible with (Q1). For the instances of each universal will, in this case, exhibit the same regularities (qua instances of that universal) in all possible worlds. Developed in this way, A+ yields a conception of universals that is similar to realist varieties of dispositional essentialism (henceforth "RDE") ${ }^{10}$, but is nevertheless distinct from such views, for reasons discussed in Section 4. Like dispositional essentialism, however, A+ is on this construal incompatible with the position of Dretske (1977), Tooley (1977), and Armstrong (1983) (henceforth "DTA"), which is committed to (Q1), since it treats the relations among universals that determine the regularities in the behavior of their instances as metaphysically contingent.

While I sympathize with critics of $(\mathrm{Q} 1)^{11}, \mathrm{~A}+$ can be made compatible with it (and thus with at least one form of quidditism, viz. [(Q1) \& (Q2) \& (Q3)]) by treating the individuative descriptions of universals it generates as non-rigid. Construed in this way, A+ implies that while universals can be distinguished by means of the regularities in the actual world that they're postulated on the basis of, a universal may also have instances in other possible worlds whose behavior has nothing in common (qua instances of that universal) with that of its actual

\footnotetext{
${ }^{9}$ I'll occasionally switch from the formal to the material mode like this, for ease of exposition. I stress, however, that $\mathrm{A}+$ is intended first and foremost as an analysis of statements about universals. It is a further question whether there are in fact universals whose nature corresponds to the content of our statements about them, as analyzed via $\mathrm{A}+$. Advocates of A+ thus have the option of holding that while A+ explicates our concept of universals, there is nothing that answers to that concept (either because one thinks that there are no universals, or that universals exist but that our concept of them somehow misrepresents their nature). To this point, whenever I switch to the material mode and speak of A+ as providing an analysis of universals, or as involving certain claims about the nature of universals, such talk should be understood as prefaced by the qualification "if universals exist and are such as our statements about them, analyzed according to A+, suggest..." Thanks to an anonymous reviewer for drawing my attention to this issue.

${ }^{10}$ See Shoemaker (1980), Fales (1990, pp.154-6), Ellis (2001, pp.127-35), and Bird (2007). Tugby (2013) argues that anyone who accepts dispositional essentialism ought to view properties as transcendent universals.

${ }^{11}$ See Shoemaker (1980), Bird (2007, pp.73-6), and Black (2000, p.94-5).
} 
instances. Insofar as DTAists are willing to allow that universals can be individuated on the basis of regularities in the behavior of their actual instances (thereby rejecting (Q2) and (Q3)), A+ may seem compatible with DTA when interpreted in this manner. As we'll see in the following section, however, A+ is inconsistent with both DTA and RDE, regardless of whether one treats the individuative descriptions of universals it generates as rigid or non-rigid.

\section{Reductive vs. non-reductive realism}

There are two different ways of analyzing statements in terms of the evidence we'd take to confirm them, one of which I'll call reductive, and the other non-reductive. These two kinds of analyses differ in whether they treat the terms that appear in the kind of statement being analyzed as referring to something distinct from (i.e. non-identical to) the evidence for such statements; non-reductive analyses do, whereas reductive analyses don't. As an example of a reductive analysis, consider behaviorist analyses of psychological statements, according to which the mental terms that appear in such statements don't refer to anything distinct from the behavior on the basis of which such statements are made. As an example of a non-reductive analysis, consider functionalist analyses of psychological statements, which treat the mental terms that appear in such statements as referring to internal states that are distinct from the sensory causes and behavioral effects in terms of which they are defined (Lewis 1972). Due to their greater simplicity, Occam's razor favors reductive analyses over non-reductive analyses, provided that the former don't render the statements they're applied to incapable of doing certain theoretical work that we want them to do. The failure of behaviorist analyses of psychological statements to satisfy this latter condition accounts for their widespread rejection in favor of functional analyses. Whenever a reductive analysis can satisfy this condition, though, parsimony gives us reason to prefer it to any corresponding non-reductive analysis. 
$\mathrm{A}+$ is a reductive analysis of statements about universals, as indicated by its insistence that such statements commit us to nothing more than the basic regularities that serve as evidence for them. However, just as behaviorists needn't be eliminativists about the mental, so too A+ needn't imply that there are no universals. Whether it does so depends on how one conceives the basic regularities that A+ analyzes universals in terms of. If one adopts what I'll call a thin conception of these regularities, which treats them in standard Humean fashion as patterns in the Humean mosaic that are described by non-modal, universally quantified sentences and consist entirely of the sum of their particular instances, then A+ leads to nominalism. Developed in this way, A+ serves as an instrument for translating statements about universals into a nominalist language, wherein talk of universals is replaced by talk of classes of particulars ${ }^{12}$ whose behavior comprises a basic thin regularity. ${ }^{13}$ For any predicate $\mathrm{F}$ that is presumed to correspond to a universal, the sentence " $x$ is F" would thus be translated roughly as follows:

"The class of Fs consists of all and only those particulars that behave in suchand-such a way \& there is no explanation for why all and only Fs behave in this way $\& x$ is one of the Fs."

Trickier sentences like " $x$ is F, G, and H, and F-ness and G-ness resemble each other" (where the predicates $\mathrm{F}, \mathrm{G}$, and $\mathrm{H}$, are all presumed to correspond to universals) might be similarly translated thus:

"There are three classes of particulars (the Fs, the Gs, and the Hs) such that, for each of these classes, all and only the members of that class behave in such-and-

\footnotetext{
${ }^{12}$ Classes here being understood as particulars that consist of (i.e. aren't distinct from and are nothing over-andabove the sum of) their members.

${ }^{13} \mathrm{~A}+$ might also give nominalists a way of distinguishing predicates that correspond to properties from predicates that don't, or of distinguishing "perfectly natural" properties (or properties that play a role similar to that which Lewis (1983) assigns to such properties) from other properties. The first two conjuncts in the following translation could be put to this use, although nominalists who have no use for these distinctions could translate " $x$ is F" simply as " $x$ is one of the Fs". More on this in Section 5.
} 
such a way \& there is no explanation for why all and only the members of each class behave in the way that they do \& the behavior exhibited by all and only the members of one of these classes (viz. the Fs) resembles the behavior exhibited by all and only the members of one of the other classes (viz. the Gs) $\& x$ is a member of all three classes."

So much for the implications of A+ when one treats the basic regularities it appeals to as thin.

There is, however, another way of conceiving the basic regularities that A+ analyzes universals in terms of, which is to view them as modal, abstract, repeatable patterns that are distinct from the sum of their particular instances, and thus something over-and-above the thin regularities that consist solely of the sum of each instance in the Humean mosaic wherein a particular behaved in such-and-such a way. Developed in tandem with this alternative, thick conception of the basic regularities it appeals to, A+ doesn't lead to nominalism, since (on this construal) to say that a particular instantiates a universal is to say that its behavior exhibits a modal, abstract, repeatable pattern that exists over-and-above the sum of its instances. ${ }^{14}$ In contrast, however, to other forms of realism (e.g. DTA and RDE), this isn't to say that the particular conforms to this pattern because it instantiates a universal, which (by virtue of its dispositional essence or relations to other universals) guarantees that each of its bearers behaves in this way. Rather, for the particular to instantiate the universal in question just is for its behavior to exhibit that pattern.

While A+ thus needn't lead to nominalism, as a reductive analysis, A+ is incompatible with non-reductive forms of realism, which treat universals as individuated in terms of, but

\footnotetext{
14 This isn't to say that nominalists can't also view basic regularities as thick. Trope-theoretic forms of dispositional essentialism might thus be seen as combining trope nominalism with a thick conception of the regularities that tropes with dispositional essences give rise to (Molnar 2003; Heil 2003, pp.111-25, 137-50; Whittle 2008). Insofar, however, as realist and trope-theoretic forms of dispositional essentialism distinguish the dispositional universals or tropes they posit from the regularities they give rise to, A+ has the advantage of simplicity over them both. See footnote 18 .
} 
nevertheless non-identical to, the regularities that serve as evidence for them. For the nonreductive realist, universals are things that determine how particulars behave while remaining distinct from the patterns they produce in their instances' behavior. In contrast, according to the reductive form of realism one gets by combining $\mathrm{A}+$ with a thick conception of the basic regularities that $\mathrm{A}+$ analyzes universals in terms of (henceforth simply "reductive realism"), we should instead think of universals as the very patterns that are exhibited in their instances' behavior. For the reductive realist, universals exist, but are reducible to basic, thick regularities in the behavior of particulars.

Conceived in this way, universals are abstract ways of behaving that are immanent in the particular instances of behavior in which they are manifested, but distinct from the sum of all such instances. Reductive realism is thus Aristotelian and constituent rather than Platonic or relational, in that universals are viewed not as existing outside of space and time, but instead as constituents of the spatio-temporally located particulars that instantiate them. ${ }^{15}$ Since not all predicates are applicable to all and only those particulars whose behavior exhibits a common basic pattern, reductive realism is also sparse. In contrast, however, to other sparse, Aristotelian, constituent forms of realism, for the reductive realist, the universals that a particular instantiates again do not determine how it behaves in virtue of their dispositional essences or relations to other universals, but are instead identical to the thick patterns manifested in its behavior. Universals are thus constituents of particulars in the same sense that a particular might be said to consist, at least partly, of what it does (and is disposed to do) over

\footnotetext{
${ }^{15}$ Realists (e.g. van Inwagen 2011) who are averse to such forms of realism might opt for a non-reductive, Platonic, relational (but still sparse) form of realism similar to the reductive form of realism described above by treating universals instead as abstract, non-spatiotemporal entities that are distinct from the basic regularities on the basis of which they're postulated, but which are related to them in something like the way that Fregean propositions are related to the particular sentences that express them. In comparison with this non-reductive, Platonic, relational alternative, however, reductive realism has the advantage of greater simplicity and can I think avoid (or at least mitigate) the problems that van Inwagen (2011) raises for constituent realism, as mentioned below.
} 
the course of its existence; its way of behaving is, we might say, part of its history, which is at least partly constitutive of the particular itself.

While this may not entirely allay the bewilderment that constituent realism produces in some opponents of the view (e.g. van Inwagen 2011, pp.393-6), reductive realists can nevertheless, I think, do more to alleviate this concern than non-reductive constituent realists (e.g. Armstrong 1983). For according to the reductive realist, to say that a universal is a constituent of a concrete particular $p$ is not to say that there is some ghostly entity that somehow worms its way into $p$, or that $p$ somehow manages to seize this ghostly entity and incorporate it into its constitution. It's merely to say that at some point during its existence, $p$ behaved (or was disposed to behave) in a way that might also be found in other particulars (or in $p$ at other times), but which is distinct from the sum of all instances of such behavior and cannot be explained in terms of any other patterns in the way things behave (Alexander 1920, pp.221-2).

In further contrast to other sparse, Aristotelian, constituent forms of realism (e.g. Armstrong's (1983, pp.96-7)), reductive realism also doesn't view universals as postulated via an inference to the best explanation for patterns in way things behave. For the reductive realist, the relation between universals and the regularities that serve as evidence for them isn't abductive in nature, for universals are identical to the thick regularities on the basis of which they're postulated, and hence cannot explain those regularities, since explanation is irreflexive. To state that a universal exists is thus merely to assert the existence of a certain sort of pattern in the behavior of particulars, not to explain that pattern. Those (e.g. van Inwagen 2011, pp.396400) who are skeptical of the use of abductive arguments in support of constituent realism should thus have no quarrel on this score with the reductive form of constituent realism that A+ yields. 
This point is worth stressing, as it gets at a fundamental difference in the motivations behind reductive and non-reductive realism. In particular, the non-reductive realist is more apt to place importance on the one over many problem, and feel a need to postulate universals to explain resemblances among particulars, or how it is that distinct particulars can be of the same type or behave in the same way. In contrast, the reductive realist is, like the nominalist, happy to treat the existence of basic regularities in the behavior of particulars as primitive and deny that the resemblances among the particulars that exhibit any such regularity requires an explanation that can be provided only by postulating a universal that they all share in common. For the reductive realist, to say that distinct particulars share a universal in common just is to say that they resemble one another by behaving in a way that cannot be explained by any other regularities. On this view, universals can't explain why distinct particulars resemble one another by behaving in the same basic manner, for universals are the thick, basic regularities that such relations of resemblance consist of. Other, non-basic regularities and corresponding relations of resemblance among particulars may be explained by universals, and thus needn't be taken as primitive, but this is simply because non-basic regularities can be explained in terms of the basic regularities that the reductive realist identifies universals with.

What, then, are the reductive realist's motivations for being a realist? If one is willing to treat basic resemblances among particulars as primitive, why not simply go nominalist? ${ }^{16}$ Here the reductive realist may cite a dissatisfaction with nominalist attempts to account for counterfactuals, chances, and nomological explanation in terms of thin regularities, thereby siding with critics of Humeanism who see such regularities as inadequate to play this role. ${ }^{17}$ It

\footnotetext{
16 Thanks to an anonymous reviewer for pressing me on this point.

17 See Dretske (1977), Tooley (1977, pp.668-71), Armstrong (1983, pp.10-68), Carroll (1994, pp.57-80), Bird (2007, pp.81-90), Briggs (2009), Lange (2013), Demarest (2017, pp.41-5), and Kimpton-Nye (2017, pp.143-7). The topics of counterfactuals and nomological explanation are taken up in the following section. Regarding chances, since reductive realists distinguish thick regularities from the sum of their instances, if they treat a given thick regularity $\mathrm{R}$ as irreducibly probabilistic (so that, e.g., any particular that manifests $\mathrm{R}$ thereby has a $50 \%$ chance of being F), they can allow the frequencies found in the sum of R's instances to deviate from the probabilities built into $\mathrm{R}$ itself (so it may turn out, e.g., that more or less than half of R's instances are actually F).
} 
is for this reason that reductive realists see fit to introduce universals (conceived as thick regularities) into their ontology: not to explain basic regularities and the resemblances among particulars that exhibit them, but rather to address the perceived failings of nominalist accounts of counterfactuals, chances, and nomological explanation. The following section takes up this issue in greater detail. For now it will suffice to note that while reductive and non-reductive realists agree that the nominalist's ontology is too impoverished to account for everything we want to account for, they differ in where precisely they see nominalism as coming up short, and their motivations for postulating universals to address these perceived inadequacies hence differ as well.

In contrast to the nominalist construal of $\mathrm{A}+$, the reductive realist version of $\mathrm{A}+$ yields the following analyses of the sentences translated above. The sentence " $x$ is F" (where the predicate $\mathrm{F}$ is presumed to correspond to a universal) becomes something like:

"There is a pattern of behavior that is exhibited by all and only those particulars we designate as Fs \& this pattern is distinct from the sum of its instances $\&$ the pattern cannot be explained by any other regularities \& $x$ is an F."

The sentence " $x$ is F, G, and H, and F-ness and G-ness resemble each other" (where the predicates $\mathrm{F}, \mathrm{G}$, and $\mathrm{H}$, are again all presumed to correspond to universals) can be similarly translated as follows:

"There are three patterns in the behavior of particulars (F, G, and $\mathrm{H})$ \& each of these patterns is distinct from the sum of its instances \& none of these patterns can be explained by any other regularities \& one of these ways of behaving (viz. F) resembles one of the others (viz. G) \& $x$ behaves in all three of these ways."

\footnotetext{
Nominalists of course have their own ways of allowing for such divergences between the chances and actual frequencies. Those dissatisfied with such stratagems may, however, find the reductive realist approach more appealing. The points mentioned in footnote 14 should be borne in mind here as well.
} 
I think reductive realism is worthy of serious consideration as a third option intermediate between the traditional alternatives of nominalism and non-reductive realism. The availability of this option means that one can agree with critics of Humeanism about the inability of thin regularities to account for counterfactuals, chances, and nomological explanation without thereby concluding that we must postulate universals in addition to the regularities that serve as evidence for them, and, conversely, that one can agree with nominalists about the explanatory idleness of universals conceived as distinct from the regularities on the basis of which they're postulated without thereby concluding that such regularities can be adequately understood in thin, Humean terms, or that everything that exists is particular. That said, I take no stance in this paper on whether A+ is best developed in the direction of nominalism or reductive realism. I only stress that there is a choice to be made here, for while A+ is consistent with nominalism, there is a form of realism that is compatible with it as well.

I $d o$ want to argue, however, that if one is going to be a realist, one ought to be a reductive realist. For while there are reasons for viewing basic regularities as thick, once one has taken this step there is, I think, no further reason to postulate universals as distinct from the regularities that serve as evidence for them. Since reductive realism is an ontologically simpler position than non-reductive realism, we ought to favor the former over the latter unless there is some theoretical work that we want our statements about universals to do that they'd be unable to do unless universals are distinguished from the regularities on the basis of which they're postulated. The next section argues that there is no such reason for paying the additional ontological cost that non-reductive realism involves.

\section{What do statements about universals commit us to?}


Why might one think that statements about universals will be unable to do certain theoretical work we want them to do if analyzed (à la $\mathrm{A}+$ ) as committing us to nothing more than the regularities that serve as evidence for them? For starters, one might suppose that universals must be distinguished from such regularities in order to explain why certain basic regularities are manifested in the behavior of some particulars but not others. In such cases, one might think that in addition to the regularity in question, we must also postulate a universal $\mathrm{U}$ that is instantiated only by those particulars whose behavior manifests that regularity, which explains why they behave in that way, whereas other particulars, which don't instantiate U, do not. The fact that the regularity is manifested only by instances of $U$ might then be explained by holding either (à la DTA) that U bears certain metaphysically contingent relations of necessitation to other universals that ensure that its instances behave in this way, or ( $a$ la RDE) that $\mathrm{U}$ is essentially such as to bestow on its instances a disposition to so behave.

I see no reason, however, why we should have to distinguish the universal ascribed to certain particulars on the basis of a regularity in their behavior from that regularity in order to explain why the regularity is manifested only by those particulars. First and foremost, it's not obvious that this fact requires an explanation. We can instead simply take it as primitive that these particulars behave in this way, while others do not. This seems all the more reasonable given that the regularities in question are presumed to be basic. For if a regularity manifested by certain particulars cannot be explained in terms of other regularities, then it shouldn't be too surprising if the fact that the regularity is manifested only by these particulars proves unexplainable as well. Moreover, even supposing that this fact does require an explanation, distinguishing universals from the regularities on the basis of which they're postulated is unlikely to be of much help, as this just moves the bump in the rug, replacing the question "Why is this regularity manifested only in the behavior of these particulars?" with the question "Why is this universal instantiated only by these particulars?" I can't think of any answer to 
the latter question that couldn't also be given (mutatis mutandis) to the former, and if one takes the latter question to be unanswerable, we would then seem better off simply taking the former fact as primitive, thereby obviating the need to distinguish universals from the regularities in their instances' behavior to explain it. ${ }^{18}$

I conclude that the fact that some regularities are manifested only in the behavior of certain particulars and not others doesn't give us any reason to distinguish universals from the regularities on the basis of which they're postulated. Is there any other theoretical work that we want universals to do that they'd be incapable of doing if analyzed along the lines of A+?

I take it that our primary purpose in postulating universals and ascribing them to particulars is to account for resemblances among numerically distinct things and distinguish classes of things that resemble one another in some significant respect from arbitrary groups, e.g. the class of things within a 2-mile radius of the Eiffel tower. A+ enables statements about universals to fulfil both these functions. According to A+, to ascribe a universal to a particular is to designate it as belonging to a class of things whose behavior exhibits the same basic pattern, so to say that two or more particulars instantiate the same universal is just another way of saying that there are certain similarities in the way they behave that cannot be explained by other regularities. Viewed in this way, the fact that certain particulars instantiate the same universal admittedly does not explain those resemblances among them that lead us to ascribe the same universal to them. For on the reductive realist version of $\mathrm{A}+$, these two facts are one and the same, while on nominalist version of $\mathrm{A}+$, there are no facts about universals, just statements that are to be translated, via A+, into statements about special classes of particulars.

\footnotetext{
${ }^{18}$ These considerations tell equally against the idea that we must postulate dispositional tropes in addition to the regularities in the behavior of the concrete particulars that possess or are constituted by them to explain why some regularities are manifested only by certain particulars. Rather than trying to explain this fact by postulating a class of perfectly resembling dispositional tropes that are only possessed by or constituents of those concrete particulars that exhibit a certain regularity (which simply raises the further question: why are these tropes only possessed by or constituents of these concrete particulars?), we can again simply take it as primitive that only these particulars behave in this way.
} 
Consequently, neither form of $A+$ can treat the fact that certain particulars exhibit the same basic regularity as analyzable in terms of the supposedly distinct, more fundamental fact that they instantiate the same universal. This doesn't imply, however, that A+ renders statements about universals incapable of accounting for resemblances among particulars. For as Lewis (1983, p.352) notes: "Not every account is an analysis!" (or at least not an analysis of the sort just mentioned, wherein the analysans is presumed to be distinct from and more fundamental than its analysandum). Advocates of A+ can instead account for the resemblances among particulars that lead us to ascribe the same universal to them by simply taking it as primitive that the same basic pattern is manifested in their behavior, which (according to A+) is all that the statement that they instantiate the same universal affirms.

The different motivations behind reductive and non-reductive realism mentioned in Section 4 come to the foreground here, as non-reductive realists likely will see the fact that certain particulars exhibit the same type of basic regularity, or resemble one another by behaving in the same basic manner, as something that requires an explanation. This explanation is provided (in their view) by postulating a universal that those particulars share in common, but which is distinct from the regularity in their behavior that it is introduced to explain (as indeed it must be, if it is to do the explanatory work assigned to it). The reductive realist, in contrast, won't see any need for an explanation to be given here; their motivations for postulating universals again lie elsewhere (e.g. in addressing perceived failings in nominalist accounts of counterfactuals, chances, and nomological explanation). One of the upshots of this paper is thus that there may still be good reasons to be a realist even if one finds the traditional motivations stemming from the one over many problem and the perceived need to explain resemblances among particulars unconvincing.

$A+$ also distinguishes arbitrary groups from classes of things that share a universal in common. According to A+, to postulate a universal is merely to say that there is a basic pattern 
in the way certain particulars behave. Where no such pattern exists in the behavior of all and only certain particulars, there is therefore no universal that all and only those particulars instantiate. A+ thus entitles us to postulate a universal of being negatively charged inasmuch as there are basic patterns in the behavior of all and only those things we designate as negatively charged. To say that there is such a universal is, according to A+, merely to assert that such patterns exist. In contrast, A+ suggests that there is no universal of being within a 2 mile radius of the Eiffel tower because there are no basic patterns in the behavior of all and only those things that are within 2 miles of the Eiffel tower. Any patterns that might be found in the behavior of such things (e.g. their ability, due to their proximity to the Eiffel tower, to reflect or emit photons that reach the Eiffel tower within a certain amount of time) can presumably be explained in terms of other, more general regularities (in this case, regularities in the speed of photons) that are also exhibited by things that are not within 2 miles of the Eiffel tower. To deny that there is a universal of being within a 2 mile radius of the Eiffel tower is thus, according to $\mathrm{A}+$, merely to assert that any patterns that might be found in the behavior of all and only those things that are within 2 miles of the Eiffel tower can be explained by other regularities in this way.

Interpreted according to $\mathrm{A}+$, statements about universals are thus able to account for resemblances between numerically distinct things and distinguish arbitrary from non-arbitrary classes, thereby fulfilling the two functions that we most want them to perform. Is there any further work that we want such statements to do for us? One might also hope to use universals to address certain questions about laws of nature, e.g. what distinguishes laws from accidental regularities? or, what enables laws to support counterfactuals and explain their instances? If (as seems plausible) basic regularities are laws, ${ }^{19}$ then it may seem circular for advocates of $\mathrm{A}+$ to

\footnotetext{
${ }^{19}$ This isn't compulsory; even those who view basic regularities as thick might deny that they're laws. Mumford (2004) thus argues that while there are necessary connections in nature (and thus thick, non-Humean patterns in the behavior of particulars), there are nevertheless, strictly speaking, no laws of nature. Another option would be
} 
appeal to universals in answering such questions, as we' $d$ then be analyzing universals in terms of laws, while at the same time analyzing laws in terms of universals. Non-reductive realists can avoid this problem by analyzing laws as relations between universals, or as derived from universals' dispositional essences, thereby treating laws as less fundamental than universals. ${ }^{20}$

My response to this worry is to deny that any circularity in A+'s treatment of laws and universals is vicious. On the contrary, we should expect our analyses of these notions to depend upon one another, for laws and universals are two sides of the same coin. Rather than treating one as more fundamental than the other, I suggest that both are equally fundamental and indeed interdefinable. To say that it is a law (as opposed to an accidental regularity) that all Fs are Gs is thus to say that the expressions " $F$ " and " $G$ " denote universals, and that the basic patterns in the behavior of Fs and Gs that lead us to interpret "F" and "G" in this way include the fact that nothing can be an $\mathrm{F}$ without also being a $\mathrm{G}$ (which fact cannot be explained by other regularities). ${ }^{21}$ Conversely, to say that " $F$ " and " $G$ " denote universals is to say that there are patterns in the behavior of particulars that we classify as Fs or Gs that cannot be explained by other regularities, and which therefore qualify as laws according to the analysis of statements about laws just described. Postulations of universals thus go hand-in-hand with the postulation of laws. Neither can be understood except in terms of the other. I'll henceforth use "A+" to

to treat laws as linguistic entities that describe certain regularities that have a special pragmatic status for us, e.g. as being particularly salient, or useful in making predictions and/or systematizing experience (Dorst 2019; Kimpton-Nye 2021). In this case, the basic regularities that reductive realists identify universals with would presumably qualify as more fundamental than the laws that (on this view) are descriptions of them, and reductive realism hence wouldn't face the circularity worry raised above. While this may consequently be an easier route for reductive realists to take in their treatment of laws, I do want to consider how the position might be developed in tandem with a more realist conception of laws, according to which laws are language-independent regularities whose status as laws doesn't depend on our pragmatic interests. Thanks to an anonymous reviewer for raising this issue.

${ }^{20}$ Kimpton-Nye (2021) argues that a version of this circularity objection actually does arise for realist advocates of what he calls "Canonical Dispositional Essentialism" (e.g. Bird 2007), because they are committed to treating laws as constituting the essences of universals, while also using universals to explain laws. (See also Jaag (2014).) If so, then so much the better for reductive realism, as RDEists of this stripe then cannot claim any advantage over reductive realism on this score. Thanks to an anonymous reviewer for pointing this out.

${ }^{21}$ I thus share Armstrong's (1983, p.162) view that "derived" laws are not genuine laws. (See footnote 26.) Some may want to add further conditions that basic regularities must satisfy in order to qualify as laws (e.g. those associated with Best Systems accounts), in which case the following sentence should be modified accordingly. 
refer to the combination of my proposed reductive analysis of statements about universals with this interdependent analysis of statements about laws.

A+ bears some similarity to the views of Cohen and Callender (2009), Loewer (2007), and Lewis (1983, p.368), who likewise hold that laws and properties are postulated together in a "package deal." 22 However, whereas these theorists are all led to an interdependent conception of laws and properties through their endorsement of a Best Systems account of laws, $\mathrm{A}+$ is neutral on the latter and arrives at the former instead through a reductive analysis of statements about universals. Further differences arise on the question of which properties figure into the package that we ought to postulate as providing the best account of the patterns we discover in the way things behave. Cohen and Callender would likely deny that there is a single such package, as (a) any set of patterns may, in their view, be accounted for by packages involving different properties, and (b) there are no "transcendent" criteria that we can use to determine which package is best independently of the properties that each postulates. Central to Cohen and Callender's argument is the idea that the only (non-interest-relative) criterion we can use to judge which package is best is the degree to which each achieves the Lewisian balance of simplicity and strength. Since this balance can only be defined relative to a particular set of properties, we cannot determine which of two packages involving different properties provides the best account of regularities that both can account for. Decisions between such packages must hence, on Cohen and Callender's view, be made solely on the basis of our present interests, and the properties that appear most relevant in light of these.

Granting that packages involving different properties are indeed incommensurable with respect to Lewisian criteria, we may still find non-arbitrary grounds for deciding between such packages by letting science make the decision for us. In contrast to Lewis' (1983, pp.346-7)

\footnotetext{
${ }^{22}$ Since these theorists speak in terms of properties instead of universals, I do the same in the next two paragraphs, to facilitate discussion of their views. This poses no problem, since A+ is compatible with treating properties as either universals or classes of resembling particulars.
} 
solution of positing an "élite minority" of special, "perfectly natural" properties that are by nature uniquely suited to figure in the package that best accounts for the patterns in the behavior of things (which, as Cohen and Callender (2009, pp.11-4) and Loewer (2007, p.322) note, raises difficulties as to how we can know that the basic kind predicates in the package we endorse correspond to the perfectly natural properties ${ }^{23}$, we might instead hold that the properties best suited for this role are just whatever properties science postulates at the limit of inquiry. Any packages remaining at that limit that differ in their kind predicates may then be treated as mere notational variants (Ladyman 2012, p.43; Worrall 2011). ${ }^{24}$ In this regard, my own view is closest to Loewer's (2007, p.324), the main difference between us being that whereas he identifies the properties in the ideal package with whatever properties will be postulated by the best final theory in fundamental physics, I see no reason to exclude the possibility that there may be patterns in the way things behave that physics cannot account for, and for which science may consequently find it necessary to postulate non-physical properties and laws. The regularities in animal behavior that we typically explain in psychological terms could turn out to be patterns of this sort.

One might worry, however, that treating our talk of laws and universals as interrelated in this way undermines our ability to practically distinguish genuine laws and universals from pseudo-universals and pseudo-laws. Granting that the circularity in A+'s treatment of laws and universals is virtuous, how do we break into this circle in any particular case? When we come across a pattern in the behavior of certain particulars, how do we determine if that pattern justifies postulating a universal that those particulars share in common, thereby treating the pattern as a law instead of an accidental regularity?

\footnotetext{
${ }^{23}$ See also Demarest (2017, pp.41-3).

${ }^{24}$ Cohen and Callender (2009, pp.6-8) would likely see this as an instance of what they call the "strategy of denial." Their objections to this strategy, however, strike me as overestimating the likelihood of substantial underdetermination at the limit of inquiry, while also underrating the option of treating any packages remaining at that limit as notational variants.
} 
The requirement that universals only be postulated on the basis of basic regularities can again be useful here. For given any pseudo-universal (e.g. grueness), any regularities that might be taken as grounds for postulating it can be explained by other regularities that give us no reason to assume that any such universal exists. The regularities that might lead one to postulate a universal of grueness thus consist entirely of such facts as that all emeralds observed thus far have been green. Given, however, the broader network of laws that we know of (which imply, e.g., that all emeralds have a similar chemical composition, that an object's chemical composition is not affected by the time at which it is observed, and that objects with similar chemical compositions have similar spectral reflectance curves and as such are typically designated as having the same color), these regularities are better explained by the supposition that all emeralds are not grue but green. ${ }^{25} \mathrm{~A}+$ 's requirement that universals be postulated only on the basis of basic regularities thus enables us to distinguish genuine universals from pseudouniversals even if universals and laws are interdefinable in the manner suggested above.

Similar considerations apply to accidental regularities, e.g. that all lumps of gold are less than 1 mile $^{3}$ in volume (Armstrong 1983, p.16). In such cases, the regularity in question can be explained by other regularities in a way that clarifies both why that regularity happens to obtain, and how it could be violated by altering certain initial conditions while leaving the basic regularities unchanged (or, to put it another way, how the regularity in question could fail to obtain in a world with the same basic regularities as the actual world). The fact that all

\footnotetext{
${ }^{25}$ What about the regularity that all emerires are grue? While this regularity can be explained by the fact that all emeralds are green and all sapphires blue, the latter regularities could in turn be explained by the fact that all emerires are grue and all sappheralds bleen. However, given again the broader network of laws that we know of (which imply, e.g., that organisms generally classify things with similar chemical compositions as being of the same type and things with very different chemical compositions as being of different types), the former regularities provide a better explanation of the latter, gruesome regularities than the other way around. One might construct gruesome parallels of these other laws as well, involving, e.g., different, appropriately gerrymandered criteria for what chemical compositions count as similar, and what counts as an organism's classifying two things as being of the same type. At the point, however, where the resulting gruesome and non-gruesome packages of laws and universals each provides an equally good explanation of the other, it seems reasonable to treat the two packages as mere notational variants in the manner suggested above (Loewer 1996, p.110).
} 
lumps of gold are less than 1 mile $^{3}$ in volume can thus be explained by other regularities (e.g. those explaining the relative abundance of different elements, and the likelihood that atoms of a given element will bond with one another), which explain both why we should expect all gold lumps to be less than 1 mile $^{3}$ in volume (given the initial conditions of the universe), and why the existence of a lump of gold greater than 1 mile $^{3}$ in volume is nevertheless not inconsistent with the basic regularities in the way that a similarly sized lump of uranium is (since gigantic lumps of gold could exist in a world with the same basic regularities as the actual world but different initial conditions, whereas no world with the same basic regularities as the actual world could contain gigantic lumps of uranium). In contrast to nomic regularities, an accidental regularity can thus be explained by other regularities that could also obtain in scenarios wherein the accidental regularity is violated (due to a change of initial conditions). ${ }^{26}$ All of this is perfectly consistent with the idea that laws and universals are interdefinable in the way A+ suggests.

A+ also explains why laws support counterfactuals. According to A+, to say that it is a law that all Fs are Gs is to say that the expressions "F" and "G" denote universals and that nothing can be an $\mathrm{F}$ without also being a $\mathrm{G}$, where this fact cannot be explained in terms of other regularities. Construed in this way, the statement "It is a law that all Fs are Gs" implies that if any non-F were an $\mathrm{F}$, then it would be a $\mathrm{G}$, for part of what we assert in making this statement is that non-G Fs are impossible. ${ }^{27}$ The notion of necessity involved in our statements about laws and the counterfactuals that such statements support may be interdefinable as well.

\footnotetext{
${ }^{26}$ We may also want to distinguish a class of regularities that are nomic but not laws; these would be regularities that can be explained in terms of basic regularities, but which could not fail to obtain in worlds with the same basic regularities as the actual world, regardless of what the initial conditions might be. The fact that all solid lumps of uranium are less than 1 mile $^{3}$ in volume might be an example.

${ }^{27}$ Reductive realism may seem better able to assign this kind of modal force to statements about laws than the nominalist version of $\mathrm{A}+$, since (in contrast to the latter) it treats the basic regularities that statements about laws are based on as thick. Those who adopt the nominalist version of A+ might hold, however, that to say that non-G Fs are impossible is merely to say that unlike a solid lump of gold greater than $1 \mathrm{mile}^{3}$ in volume, there could be no non-G F unless some basic regularity were violated, which is compatible with claiming that all regularities are merely thin.
} 
Pace non-reductive realists, rather than treating certain primitive modal connections or relations of necessitation between universals as explaining (and thus as distinct from and more fundamental than) the counterfactuals that a given law supports, any modal facts that we take the statement "It is a law that all Fs are Gs" to imply may simply be equivalent to the counterfactual statement that if any non-F were an $\mathrm{F}$, then it would be a G. To say that $\mathrm{F}$ "necessitates" G, or that non-G Fs are impossible, or that if some non-F were an F, then it would be a G, may thus all be different ways of asserting the same thing.

A+ also explains why laws explain their instances. This is again owing to its analysis of statements of the form "It is a law that all Fs are Gs" as asserting (among other things) that nothing can be an F without being a G. Construed in this way, such statements imply that nonG Fs are impossible, which explains both why any non-F would be a $\mathrm{G}$ if it were an $\mathrm{F}$, and why any given $\mathrm{F}$ is also a $\mathrm{G}$. The ability of laws to support counterfactuals thus goes hand-in-hand with their ability to explain their instances. Both derive from the interdependent nature of laws and universals, and the consequent fact that in ascribing a universal to a particular, our ascription often carries further implications as to what other universals that particular or certain other particulars do (or don't) instantiate (where the instantiation of these universals again consists solely in the existence of certain patterns in the way things behave).

\section{Conclusion}

Analyzed according to A+, statements about universals thus seem capable of doing all the theoretical work that we most want them to do. Even if interpreted as asserting nothing more than the existence of basic patterns in the behavior of particulars, such statements still enable us to account for resemblances between numerically distinct things, distinguish laws and universals from pseudo-universals and accidental regularities, and explain why laws 
support counterfactuals and explain their instances. There is consequently no reason to interpret statements about universals as committing us to anything beyond the existence of such patterns, as A+ suggests. I therefore recommend the adoption of this analysis as a useful way of understanding what we're talking about when we speak of universals. ${ }^{28}$

\footnotetext{
${ }^{28}$ Many thanks to two anonymous reviewers for their insightful comments, and to participants in the 2019 PHYSIS Conference on The Problem of Universals for their helpful feedback on an early version of this paper. Special thanks also to Jim Levine for a conversation that provided the initial impetus for this paper, and to Alison Fernandes for several conversations that were extremely helpful in clarifying my thoughts on these issues, and (for me, at least) highly enjoyable as well.
} 


\section{Works Cited}

Alexander, Samuel. 1920. Space, Time, and Deity, vol. 1. Macmillan.

Armstrong, David. 1983. What is a Law of Nature? Cambridge University Press.

Ayer, A.J. 1936. Language, Truth, and Logic. Penguin Books.

Berkeley, George. 1710/1982. A Treatise Concerning the Principles of Human Knowledge.

Hackett Publishing Company.

Bird, Alexander. 2007. Nature's Metaphysics. Oxford University Press.

Black, Robert. 2000. "Against Quidditism.” Australasian Journal of Philosophy 78: 87-104.

Briggs, Rachael. 2009. "The Anatomy of the Big Bad Bug." Nous 43: 428-49.

Carnap, Rudolf. 1932. "Psychology in Physical Language." Erkenntnis 3: 107-42.

Carroll, John. 1994. Laws of Nature. Cambridge University Press.

Cohen, Jonathan, and Craig Callender. 2009. "A Better Best System Account of Lawhood." Philosophical Studies 145: 1-34.

Demarest, Heather. 2017. "Powerful Properties, Powerless Laws." In Causal Powers, ed. Jacobs. Oxford University Press.

Dorst, Chris. 2019. "Towards a Best Predictive System Account of Laws of Nature." British Journal for the Philosophy of Science 70: 877-900.

Dretske, Fred. 1977. "Laws of Nature." Philosophy of Science 44: 248-68.

Ellis, Brian. 2001. Scientific Essentialism. Cambridge University Press.

Fales, Evan. 1990. Causation and Universals. Routledge.

Heil, John. 2003. From an Ontological Point of View. Oxford University Press.

Hume, David. 1748/2007. An Enquiry Concerning Human Understanding. Cambridge University Press.

Jaag, Siegfried. 2014. "Dispositional Essentialism and the Grounding of Natural Modality." Philosophers' Imprint 14: 1-21.

Kim, Jaegwon. 1992. "Multiple Realization and the Metaphysics of Reduction." Philosophy and Phenomenological Research 52: 1-26.

Kimpton-Nye, Samuel. 2017. "Humean Laws in an unHumean World." Journal of the American Philosophical Association 3: 129-47.

. 2021. "Reconsidering the Dispositional Essentialist Canon." Philosophical Studies https://doi.org/10.1007/s11098-021-01607-2.

Ladyman, James. 2012. "Science, Metaphysics and Method." Philosophical Studies 160: 31-51.

Lange, Marc. 2013. "Grounding, Scientific Explanation, and Humean Laws." Philosophical Studies 164: 255-261.

Lewis, David. 1972. "Psychophysical and Theoretical Identifications." Australasian Journal of Philosophy 50: 249-58.

-1983. "New Work for a Theory of Universals." Australasian Journal of Philosophy 61: 343-77.

Loewer, Barry. 1996. "Humean Supervenience." Philosophical Topics 24: 101-27.

- 2007. "Laws and Natural Properties." Philosophical Topics 35: 313-28.

Molnar, George. 2003. Powers: A Study in Metaphysics. Oxford University Press.

Mumford, Stephen. 2004. Laws in Nature. Routledge.

Russell, Bertrand. 1914. "The Relation of Sense-Data to Physics." Scientia 16: 1-27.

Shoemaker, Sydney. 1980. "Causality and Properties." In Time and Cause, ed. van Inwagen. Springer.

Tooley, Michael. 1977. “The Nature of Laws.” Canadian Journal of Philosophy 7: 667-98.

Tugby, Matthew. 2013. "Platonic Dispositionalism.” Mind 122: 451-80. 
van Inwagen, Peter. 2011. "Relational vs. Constituent Ontologies." Philosophical Perspectives 25: 389-405.

Whittle, Ann. 2008. "A Functionalist Theory of Properties." Philosophy and Phenomenological Research 77: 59-82.

Worrall, John. 2011. "Underdetermination, Realism and Empirical Equivalence." Synthese 180: $157-72$. 